\title{
Architecture shapes contractility in actomyosin networks
}

Gijsje H. Koenderink ${ }^{1}$ and Ewa K. Paluch ${ }^{2}$

${ }^{1}$ AMOLF, Biological Soft Matter group, Science Park 104, 1098 XG Amsterdam, the Netherlands

${ }^{2}$ MRC Laboratory for Molecular Cell Biology, University College London, London WC1E 6BT, United Kingdom

e.paluch@ucl.ac.uk and g.koenderink@amolf.nl

Word count: 2532 words (not including abstract)

Short title (55 characters):

Actin network architecture and contractility 


\begin{abstract}
Myosin-driven contraction of the actin cytoskeleton is at the base of cell and tissue morphogenesis. At the molecular level, myosin motors drive contraction by sliding actin filaments past one another using energy produced by ATP hydrolysis. How this microscopic sliding activity gives rise to cell-scale contractions has been an active research question first in muscle cells, and over the last few decades in non-muscle cells. While many early investigations focused on myosin motor activity, increasingly, the nanoscale architecture of the actin network emerges as a key regulator of contractility. Here we review theoretical and in vitro reconstitution studies that have uncovered some of the key mechanisms by which actin network organization controls contractile tension generation. We then discuss recent findings indicating that similar principles apply in cells.
\end{abstract}




\section{Introduction:}

Actomyosin contractility is at the base of cellular morphogenesis and mechanosensing. Shape changes of animal cells as they divide, migrate, or form tissues in developing embryos, are driven by gradients in actomyosin contractility (reviewed in [1,2]). Furthermore, contractile forces exerted on neighboring cells and the extracellular matrix allow cells to sense the stiffness of their environment, which influences cell migration, cell shape dynamics within tissues, as well as cellular fate decisions (reviewed in [3]).

The mechanisms of actomyosin contractility were first investigated in striated muscle cells [4]. In these cells, actomyosin is arranged in highly ordered, one-dimensional arrays called sarcomeres, where network architecture is perfectly adapted for contractility generation (Fig. 1a). In non-muscle cells, actomyosin structures are generally less ordered, ranging from stress fibers, where actin filaments are bundled but lack clear polarity, to the cellular cortex, where actin forms a mostly isotropic network (Fig. 1b). In such disordered structures, contractile tension generation cannot be understood in terms of a sarcomeric mechanism. Partly due to the difficulty in obtaining information on the spatial organization and dynamics of actin filaments in cellular networks, the mechanisms controlling actomyosin tension generation in nonmuscle cells have long remained elusive. In fact, in morphogenesis studies, cortical contractile tension has often been assumed to be simply proportional to the levels of myosin II at the cortex [5-8], with little attention paid to the organization of the actin network itself.

Yet, in vitro studies of cell-free actomyosin systems reconstituted from purified components, as well as theoretical models clearly indicate that the spatial arrangement and physical properties of the actin filaments in the network are, alongside myosin motors, key to tension regulation (reviewed in [9]). Several recent studies in cells indeed indicate that changing actin network organization can strongly affect contractile tension even when myosin activity remains unchanged [10,11]. Increasingly, the nanoscale architecture of the actin network emerges as a key regulator of contractility both in vitro and in vivo. 
We review here in vitro and theoretical studies demonstrating that different aspects of actin network architecture are key regulators of contractile tension generation. We also discuss recent findings indicating that similar principles apply in cells. Finally, we highlight some of the important open questions in the field.

\section{Tension generation: why do contractile forces dominate?}

Actomyosin contractility predominantly results from the mechanical action of myosin II motors, which use energy released from ATP hydrolysis to exert forces on actin. Actin filaments (F-actin) have structural polarity conferred by the head-to-tail assembly of the actin monomers, leading to two distinct filament ends denoted as the minus (or pointed) and plus (or barbed) ends. Myosin II motors have two globular head domains joined by a long tail domain. The head domains bind to actin filaments and selectively move towards the plus ends, while the tail domains serve to assemble myosin II molecules into bipolar filaments with motor heads on the two ends and tails packed in the center. This bipolar architecture allows myosin filaments to slide antiparallel actin filaments with respect to each other. Depending on the arrangement of the actin filaments, the sliding activity can give rise to either a contractile or an extensile force (Fig. 2a). Yet, all known cellular actomyosin structures are overall contractile, indicating that some physical bias results in contractile forces dominating.

In skeletal muscle, this bias unequivocally originates from the arrangement of actin and myosin in repeating aligned arrays called sarcomeres, where myosin bipolar filaments are localized in between antiparallel actin filaments having their minus ends inwards and their plus ends outwards and anchored at Z-disc regions in between sarcomeres (Fig. 1a). The localization of the myosin clusters in the vicinity of F-actin minus ends and the co-alignment of myosin and actin filaments convert the sliding activity of the motors into uniform contraction [12]. Some actin structures in nonmuscle cells such as stress fibers and contractile structures associated with integrinbased adhesions display some level of sarcomeric order, but with varied polarity patterns $[13,14]$. In strong contrast, the actomyosin cortex lacks any apparent order, making contractile and extensile actomyosin configurations equally likely (Fig. 2a) [15]. The search for mechanisms that bias isotropic actomyosin networks towards 
contraction has been an active research focus for both experimentalists and theorists for the last two decades.

Broadly speaking, the mechanisms proposed so far ascribe the bias towards contractility either to mechanical asymmetries or to self-organization of contractile force dipoles (Fig. 2 b,c). Most mechanical models attribute contractility to the nonlinear elastic properties of actin filaments. Since actin filaments are semi-flexible with a persistence length around $10 \mu \mathrm{m}$ [16], they strongly resist tension but filament portions longer than $\sim 0.3 \mu \mathrm{m}$ readily buckle under compressive forces comparable to those generated by single molecular motors $(\sim 2 \mathrm{pN})$. Buckling relaxes extensile configurations, and can thus bias actomyosin bundles and networks towards contraction (Fig. 2b) [17,18]. An important prerequisite is that forces can propagate across the system, meaning that the actin filaments need to be crosslinked. Strong experimental support for this mechanism comes from direct observations of buckling of individual fluorescently labeled actin filaments during contraction of disordered bundles and 2D networks in vitro $[17,19,20]$. The degree of shortening of filaments by buckling correlates with the macroscopic shrinkage of the networks, further supporting the buckling model $[19,21]$. Buckling can sometimes lead to filament severing, which further promotes contraction [19].

Experimental evidence of a buckling mechanism promoting contractility in cells is at this point missing. A potentially crucial difference between cellular networks like the cortex and in vitro biomimetic systems is the length of the actin filaments between crosslinking points. In biomimetic assays filaments tend to be longer than the persistence length and the distance between adjacent crosslinks is on the order of 1 $\mu \mathrm{m}$ [22]. In contrast, recent work suggests that the cortex is made of filaments much shorter than the persistence length and contains a mixture of formin-nucleated filaments with lengths on the order of $1 \mu \mathrm{m}$, and much shorter Arp2/3-nucleated filaments in the $100 \mathrm{~nm}$ range [23]. Furthermore, typical cortical mesh sizes range between 30 and $200 \mathrm{~nm}$, suggesting very short distances between crosslinkers [24,25]. Actin filament segments between crosslinking points may therefore be too short and rigid for buckling to strongly contribute to contractility generation in cellular networks. 
Self-organized polarity has been proposed as an alternative contractility-promoting mechanism. The best-studied example is motor-driven polarity sorting of actin filaments. The basic idea is that if motors processively walk along actin filaments and slow down or stall before they detach from the plus end, they can cause polarity sorting of the filaments into radial arrays referred to as asters, connected by cables of antiparallel actin filaments that resemble sarcomere-like contractile structures [26] (Fig. 2c). Theoretically, polarity sorting could also be achieved via actin treadmilling: if actin filament plus end elongation and minus end shrinkage are faster than myosin movement, treadmilling can bias myosin to F-actin minus ends, which is a contractile configuration similar to that in sarcomeres [27] (Fig. 2c).

In networks, a clear signature of polarity sorting should be the formation of radial arrays of filaments (asters), where the filament ends point inwards and motors accumulate in the center. In vitro, such aster-like arrangements have been observed both in microtubule-motor mixtures [28] and in actomyosin networks [29-31]. In many cell types, the cortex also tends to form aster-like actin structures and compact myosin foci. However, it is unclear to what extent these are due to polarity sorting, or to other patterning processes driven by actin nucleation or RhoA-mediated regulation of myosin [32-34].

In addition to these mechanisms, simulations suggest that the finite size of myosin bipolar filaments may promote contraction by favoring rotation of myosin filaments toward low-energy contractile configurations [35,36] or by generating anisotropic forces by myosin heads at the filament ends [37]. To our knowledge, these predictions have not been tested experimentally.

\section{Network architecture and tension: the importance of being well connected}

Though it remains unclear which of the mechanisms described above determines the bias towards contractility in cellular networks, network connectivity via actin crosslinkers is a key determinant in all current models (Fig. 2). Interestingly, recent work highlights that further to promoting contractility, crosslinking also tunes the length scale of contractions and the magnitude of the stresses developed. 
Cell-free experiments demonstrate that long-range coordinated contraction only occurs above a threshold connectivity provided by crosslinks [38]. This threshold was identified as the percolation threshold, where all filaments are connected so that motor-driven stresses propagate across the entire system [38,39]. Below the percolation threshold, myosins contract the network only on short length scales, creating small clusters [31,38]. In cellular actomyosin networks, the role of crosslinking in actomyosin tension generation is harder to investigate because of redundancies between the vast array of different crosslinkers present. Nonetheless, several recent studies have highlighted the importance of crosslinking for cellular contractility. Experiments in C. Elegans embryos showed that crosslinking by plastin is required for effective long-range cortex contraction [11]. Similarly, depletion of the actin bundling protein Eps8 decreases cortical tension in cancer cells [40], and depletion of different cross-linkers, including alpha-actinin and fascin, decreases cellular rounding force, a readout of tension, in mitotic cells [41].

Interestingly, excessive crosslinking can be counter-productive and limit contractile tension generation. Early experiments investigating bulk contractility in in vitro actomyosin networks have already shown that a minimal level of cross-linking is required for contraction, but that excessive cross-linking is detrimental [42]. More recent theoretical and in vitro work has further dissected the relationship between crosslinking, motor activity and network contractility $[39,43,44]$. The emerging view is that maximal contractile tension is achieved at an intermediate level for crosslinking. Consistent with this idea, a recent study combining experiments on the cellular cortex and simulations suggests that cortical tension is maximum at intermediate actin filament lengths [10]. This tension optimum likely results from intermediate connectivity favoring contractility build up, as at a given crosslinker density, filament length directly correlates with network connectivity.

How exactly excessive connectivity limits contractility is not well understood. One possible explanation is that at intermediate connectivity, the network can remodel in response to myosin-mediated stresses, and that such remodeling favors contractile configurations [10]. In contrast, remodeling is limited in an overly connected network, leading to lower tension. Network remodeling could promote contractile tension by mechanisms like polarity sorting (Fig. 2c), or by the relaxation of extensile 
stresses by a buckling-like behavior of local network configurations. While these are plausible mechanisms, they remain speculative largely due to the difficulty in obtaining microscopic data on the dynamics of single actin and myosin filaments in dense contractile networks, particularly in cells.

In cells, connectivity is modulated by network turnover. Crosslinkers and actin filaments turn over within seconds and tens of seconds respectively [1]. Microscopic simulations indicate that maximum tension is expected for an optimum ratio of the filament and cross-linker turnover rates [36]. Theory and experiments further suggest that turnover could deeply affect network behavior. Turnover is thought to stabilize dynamic contractile steady states with a homogeneous myosin distribution, as opposed to irreversible contraction into clusters displayed by networks that lack turnover [43,45-47]. Interestingly, a number of models indicate that turnover should promote cyclic contractions, where the actomyosin network continuously pulses $[44,48,49]$. Such pulsed contractions appear to be very common in cell and tissue morphogenesis (reviewed in [2]).

Mechanosensitive modulation of bond lifetimes could also affect network connectivity and tension. For instance, in in vitro actin networks crosslinked with fascin, myosin was shown to cause crosslink unbinding at high motor densities [38]. Rather than being prevented from contracting at high crosslinking levels, the network was actively ruptured into clusters. Intriguingly, while fascin appears to form slip bonds, whose lifetime decreases with loading so fascin is depleted from regions of high contractile stress [38], other cross-linkers, like $\alpha$-actinin- 4 , form catch bonds and accumulate in stressed regions [50]. Mechanical stress has also been shown to affect the rates of formin-based actin filament elongation, with load making elongation faster for the mammalian Dial and budding yeast Bni1 formins [51,52], and slower for the fission yeast formin Cdc12 [53]. Myosin II motors themselves also form catch bonds [54]. How mechanosensitive effects influence tension generation has not been systematically investigated.

Finally, in addition to the level of connectivity, the specific geometric arrangement of actin networks could also influence tension. An interesting recent in vitro study used 
surface micropatterning to impose actin filament arrangement into either bundled or branched structures with disordered (mixed polarity) or ordered (antiparallel) filaments [39]. Upon exposure to myosin motors, Arp2/3-mediated branching appeared to promote contractility the most, possibly by providing more efficient percolation than bundling proteins. In contrast, several studies suggest that in cells, Arp2/3 acts to reduce contractile tension at the cortex [55]. Decreasing Arp2/3 activity promotes the formation of cellular blebs, protrusions that grow because of cortex contractions [55-57], and enhances myosin II-powered retrograde flow in neuronal growth cones [58]. These observations suggest that Arp2/3-nucleated actin networks may not provide an optimal scaffold for tension generation at the cell cortex. However, the microscopic basis of Arp2/3 effect on tension remains unclear, as information on cortical actin filament arrangement is still very limited (reviewed in (Chugh and Paluch, submitted)). 


\section{Conclusions and open questions}

The last decade has seen considerable progress in our understanding of contractility in non-sarcomeric actomyosin networks. In vitro assays and modeling have been particularly crucial in identifying the basic mechanisms of tension generation in a disordered network. Recent studies in vitro but also in cells have clearly shown that the nanoscale architecture of the actin network is a crucial determinant of tension, and that the level of crosslinking is a particularly important parameter. While we focused here on the actin cortex, cell contractility mediated by stress fibers also appears to depend on the architecture and connectivity of the fiber network [59],. Recent work also suggests that at a supracellular level, actomyosin networks architecture can be tuned in response to mechanical constraints to control force orientation during tissue morphogenesis [60].

Despite an increasingly refined understanding of contractility in vitro and despite detailed knowledge of the molecular composition of the actomyosin 'contractome' [61], the physical mechanisms controlling contractility in cells, particularly at the actomyosin cortex, remain insufficiently understood. This is partly due to inherent limitations of in vitro studies and modeling, where network components are usually restricted to actin, myosin and crosslinkers. Such reductionist approaches are powerful, as network composition is fully tractable, making predictions quantitative and precisely testable. However, such approaches can of course only unveil mechanisms relying on the specific components investigated. Cellular networks typically contain $>100$ regulatory proteins [62], many of which directly or indirectly affect tension [41].

Another key challenge is the difficulty in uncovering the organization and dynamics of actin filaments and actin-binding proteins in dense actomyosin networks. Super resolution microscopy and improvements in electron microscopy are starting to overcome this limitation. Recent studies have for example successfully used advanced image analysis and super-resolution microscopy to beat the diffraction limit and investigate the thickness and organization of the cellular cortex $[10,63]$, or to follow myosin minifilaments during cortex contractions $[64,65]$. Dissecting the nanoscale 
architecture of cellular actin networks and investigating how they dynamically remodel as cells contract and deform constitutes an exciting future research avenue.

\section{Acknowledgements}

G.H.K. gratefully acknowledges support by the European Research Council (Starting Grant 335672-MINICELL). E.K.P. gratefully acknowledges funding from the Medical Research Council UK (MRC Programme Award MC_UU_12018/5) and the European Research Council (Starting Grant 311637-MorphoCorDiv). 


\section{Figure legends:}

\section{Figure 1: Actomyosin networks in cells.}

a) In muscle cells, actin and myosin are organized into sarcomeres. The ordered organization of a sarcomere promotes contractility. Top: image of sarcomeres in Drosophila flight muscle; red: actin (phalloidin), green: the myosin-rod-associated protein flightin-GFP, image obtained with permission from [66]. Bottom: schematic of the organization of a sarcomere. b) In non-muscle cells actomyosin networks are less ordered. Cell images, kindly provided by Murielle Serres (Paluch lab), represent HeLa cells in interphase and mitosis, where DNA (red) and F-actin (cyan) have been labeled. Scale bars: $10 \mu \mathrm{m}$.

\section{Figure 2: Tension generation in isotropic actomyosin networks.}

a) In a disordered network, contractile and extensile actomyosin configurations are equally likely. b) Filament buckling can relax extensile forces and bias the network force distribution towards contraction. c) Under certain kinetic conditions, myosin minifilament processive walking or actin turnover could bias networks towards contractile configurations (details in text). 


\section{References}

1. Salbreux G, Charras G, Paluch E: Actin cortex mechanics and cellular morphogenesis. Trends Cell Biol 2012, 22:536-545.

2. Levayer R, Lecuit $\mathrm{T}$ : Biomechanical regulation of contractility: spatial control and dynamics. Trends Cell Biol 2011.

3. Heisenberg CP, Bellaiche Y: Forces in tissue morphogenesis and patterning. Cell 2013, 153:948-962.

4. Huxley HE: A personal view of muscle and motility mechanisms. Annu Rev Physiol 1996, 58:1-19.

5. Bergert M, Erzberger A, Desai RA, Aspalter IM, Oates AC, Charras G, Salbreux $\mathrm{G}$, Paluch EK: Force transmission during adhesion-independent migration. Nat Cell Biol 2015, 17:524-529.

6. Turlier H, Audoly B, Prost J, Joanny JF: Furrow constriction in animal cell cytokinesis. Biophys $J$ 2014, 106:114-123.

7. Mayer M, Depken M, Bois J, Jülicher F, Grill SW: Anisotropies in cortical tension reveal the physical basis of cortical flow in polarising $\mathrm{C}$. elegans zygotes. Nature 2010, 467:617-621.

8. Machado PF, Duque J, Etienne J, Martinez-Arias A, Blanchard GB, Gorfinkiel N: Emergent material properties of developing epithelial tissues. $B M C \mathrm{Biol}$ 2015, 13:98.

9. Murrell M, Oakes PW, Lenz M, Gardel ML: Forcing cells into shape: the mechanics of actomyosin contractility. Nat Rev Mol Cell Biol 2015, 16:486498.

10. Chugh P, Clark AG, Smith MB, Cassani DAD, Dierkes K, Ragab A, Roux PP, Charras G, Salbreux G, Paluch EK: Actin cortex architecture regulates cell surface tension. Nat Cell Biol 2017, 19:689-697.

**

This paper investigates the role of actin cortex organization in the control of cortical tension. It shows that proteins that affect actin cortex thickness also affect cortical tension, even though myosin activity appears unchanged. It concludes that changing structural parameters of actin network architecture, such as actin filament length, can modulate actomyosin tension.

11. Ding WY, Ong HT, Hara Y, Wongsantichon J, Toyama Y, Robinson RC, Nedelec F, Zaidel-Bar R: Plastin increases cortical connectivity to facilitate robust polarization and timely cytokinesis. J Cell Biol 2017, 216:1371-1386.

**

This paper demonstrates in C. Elegans embryos that crosslinking by the conserved actin-binding protein plastin is required for effective long-range contraction of the actin-myosin cortex. This finding strongly suggests the existence of a percolation threshold for cortical contractility in cells, consistent with in vitro studies and theoretical models of active gels.

12. Gautel M, Djinovic-Carugo K: The sarcomeric cytoskeleton: from molecules to motion. J Exp Biol 2016, 219:135-145.

13. Burridge K, Wittchen ES: The tension mounts: stress fibers as forcegenerating mechanotransducers. J Cell Biol 2013, 200:9-19. 
14. Wolfenson H, Meacci G, Liu S, Stachowiak MR, Iskratsch T, Ghassemi S, RocaCusachs P, O'Shaughnessy B, Hone J, Sheetz MP: Tropomyosin controls sarcomere-like contractions for rigidity sensing and suppressing growth on soft matrices. Nat Cell Biol 2016, 18:33-42.

15. Clark Andrew G, Wartlick O, Salbreux G, Paluch Ewa K: Stresses at the Cell Surface during Animal Cell Morphogenesis. Current Biology 2014, 24:R484-R494.

16. Kang H, Bradley MJ, McCullough BR, Pierre A, Grintsevich EE, Reisler E, De La Cruz EM: Identification of cation-binding sites on actin that drive polymerization and modulate bending stiffness. Proc Natl Acad Sci U S A 2012, 109:16923-16927.

17. Lenz M, Thoresen T, Gardel ML, Dinner AR: Contractile units in disordered actomyosin bundles arise from F-actin buckling. Phys Rev Lett 2012, 108:238107.

18. Ronceray P, Broedersz CP, Lenz M: Fiber networks amplify active stress. Proc Natl Acad Sci U S A 2016, 113:2827-2832.

$* *$

This paper presents a general theoretical model of the transmission of forces in disordered fiber networks generated by an active unit such as a molecular motor. The work shows that irrespective of whether the active unit is contractile or extensile, the disordered network structure results in isotropic contraction due to collective fiber buckling in the vicinity of the active unit.

19. Murrell MP, Gardel ML: F-actin buckling coordinates contractility and severing in a biomimetic actomyosin cortex. Proc Natl Acad Sci U S A 2012, 109:20820-20825.

20. Vogel SK, Petrasek Z, Heinemann F, Schwille P: Myosin motors fragment and compact membrane-bound actin filaments. Elife 2013, 2:e0116.

21. Linsmeier I, Banerjee S, Oakes PW, Jung W, Kim T, Murrell MP: Disordered actomyosin networks are sufficient to produce cooperative and telescopic contractility. Nat Commun 2016, 7:12615.

22. MacKintosh FC, Levine AJ: Nonequilibrium mechanics and dynamics of motor-activated gels. Phys Rev Lett 2008, 100:018104.

23. Fritzsche M, Erlenkamper C, Moeendarbary E, Charras G, Kruse K: Actin kinetics shapes cortical network structure and mechanics. Sci $A d v 2016$, 2:e1501337.

24. Fujiwara TK, Iwasawa K, Kalay Z, Tsunoyama TA, Watanabe Y, Umemura YM, Murakoshi H, Suzuki KG, Nemoto YL, Morone N, et al.: Confined diffusion of transmembrane proteins and lipids induced by the same actin meshwork lining the plasma membrane. Mol Biol Cell 2016, 27:1101-1119.

25. Bovellan M, Romeo Y, Biro M, Boden A, Chugh P, Yonis A, Vaghela M, Fritzsche M, Moulding D, Thorogate R, et al.: Cellular control of cortical actin nucleation. Curr Biol 2014, 24:1628-1635.

26. Kruse K, Julicher F: Actively contracting bundles of polar filaments. Phys Rev Lett 2000, 85:1778-1781.

27. Oelz DB, Rubinstein BY, Mogilner A: A Combination of Actin Treadmilling and Cross-Linking Drives Contraction of Random Actomyosin Arrays. Biophys J 2015, 109:1818-1829.

28. Foster PJ, Furthauer S, Shelley MJ, Needleman DJ: Active contraction of microtubule networks. Elife 2015, 4. 
29. Soares e Silva M, Depken M, Stuhrmann B, Korsten M, MacKintosh FC, Koenderink GH: Active multistage coarsening of actin networks driven by myosin motors. Proc Natl Acad Sci U S A 2011, 108:9408-9413.

30. Koster DV, Husain K, Iljazi E, Bhat A, Bieling P, Mullins RD, Rao M, Mayor S: Actomyosin dynamics drive local membrane component organization in an in vitro active composite layer. Proc Natl Acad Sci U S A 2016, 113:E1645-1654.

31. Stam S, Freedman SL, Banerjee S, Weirich KL, Dinner AR, Gardel ML: Filament rigidity and connectivity tune the deformation modes of active $*$ biopolymer networks. Proc Natl Acad Sci U S A 2017, 114:E10037-E10045.

This paper combines in vitro experiments and computer simulations to investigate how stiffness and connectivity control myosin-generated stresses. It shows that for rigid filament bundles, increasing network connectivity results in a transition between extensile and contractile deformations.

32. Fritzsche M, Li D, Colin-York H, Chang VT, Moeendarbary E, Felce JH, Sezgin E, Charras G, Betzig E, Eggeling C: Self-organizing actin patterns shape membrane architecture but not cell mechanics. Nat Commun 2017, 8:14347.

33. Nishikawa M, Naganathan SR, Julicher F, Grill SW: Controlling contractile instabilities in the actomyosin cortex. Elife 2017, 6.

34. Coravos JS, Martin AC: Apical Sarcomere-like Actomyosin Contracts Nonmuscle Drosophila Epithelial Cells. Dev Cell 2016, 39:346-358.

$*$

This paper demonstrates that actin architecture is a key regulator of contractility of the apical cortex in Drosophila epithelial cells. It shows that actin filament plus ends are enriched on the sides of the contracting apex, whereas myosins localize towards the middle, suggesting that actomyosin is organized in a radial sarcomere-like structure during apex contractions. Centrally localized ROCK activity controls this localization and is essential for effective contraction.

35. Dasanayake NL, Carlsson AE: Stress generation by myosin minifilaments in actin bundles. Phys Biol 2013, 10:036006.

36. Hiraiwa T, Salbreux G: Role of Turnover in Active Stress Generation in a Filament Network. Phys Rev Lett 2016, 116:188101.

37. Rubinstein BY, Mogilner A: Myosin Clusters of Finite Size Develop Contractile Stress in 1D Random Actin Arrays. Biophys J 2017, 113:937947.

38. Alvarado J, Sheinman M, Sharma A, MacKintosh FC, Koenderink GH: Molecular motors robustly drive active gels to a critically connected state. Nat. Phys. 2013, 9:591-597.

39. Ennomani H, Letort G, Guerin C, Martiel JL, Cao W, Nedelec F, De La Cruz EM, Thery M, Blanchoin L: Architecture and connectivity govern actin network contractility. Curr Biol 2016, 26:616-626.

$* *$

This paper uses an original actomyosin reconstitution approach based on surface micropatterning to control network geometry and architecture and test their effects on contractility. Combining experiments and simulations, the paper shows that 
contractility is regulated by network connectivity, which appears higher for branched than for crosslinked networks.

40. Logue JS, Cartagena-Rivera AX, Baird MA, Davidson MW, Chadwick RS, Waterman CM: Erk regulation of actin capping and bundling by Eps8 promotes cortex tension and leader bleb-based migration. Elife 2015, 4:e08314.

41. Toyoda Y, Cattin CJ, Stewart MP, Poser I, Theis M, Kurzchalia TV, Buchholz F, Hyman AA, Muller DJ: Genome-scale single-cell mechanical phenotyping reveals disease-related genes involved in mitotic rounding. Nat Commun 2017, 8:1266.

$* *$

This paper reports an AFM-based genome-scaled screen of the regulation of mitotic rounding forces, a readout of mitotic cortex tension. It identifies $\sim 50$ key regulators, many of which were not previously connected to cell mechanics. It provides an important resource for groups investigating cortex tension.

\section{Janson LW, Kolega J, Taylor DL: Modulation of contraction by} gelation/solation in a reconstituted motile model. J Cell Biol 1991, 114:1005-1015.

43. Bendix PM, Koenderink GH, Cuvelier D, Dogic Z, Koeleman BN, Brieher WM, Field CM, Mahadevan L, Weitz DA: A quantitative analysis of contractility in active cytoskeletal protein networks. Biophys $J$ 2008, 94:3126-3136.

44. Belmonte JM, Leptin M, Nedelec F: A theory that predicts behaviors of disordered cytoskeletal networks. Mol Syst Biol 2017, 13:941.

$*$

The authors present the first analytical model for isotropic filament-motor networks that predicts under which conditions isotropic networks are contractile or expansile. The model offers a unifying framework for the investigation of self-organization mechanisms of networks of cytoskeletal filaments, including actin.

45. Vavylonis D, Wu JQ, Hao S, O'Shaughnessy B, Pollard TD: Assembly mechanism of the contractile ring for cytokinesis by fission yeast. Science 2008, 319:97-100.

46. Chen Q, Pollard TD: Actin filament severing by cofilin is more important for assembly than constriction of the cytokinetic contractile ring. $J$ Cell Biol 2011, 195:485-498.

47. Abu Shah E, Keren K: Symmetry breaking in reconstituted actin cortices. Elife 2014, 3:e01433.

48. Mak M, Zaman MH, Kamm RD, Kim T: Interplay of active processes modulates tension and drives phase transition in self-renewing, motordriven cytoskeletal networks. Nat Commun 2016, 7:10323.

49. Sedzinski J, Biro M, Oswald A, Tinevez JY, Salbreux G, Paluch E: Polar actomyosin contractility destabilizes the position of the cytokinetic furrow. Nature 2011, 476:462-466.

50. Schiffhauer ES, Luo T, Mohan K, Srivastava V, Qian X, Griffis ER, Iglesias PA, Robinson DN: Mechanoaccumulative Elements of the Mammalian Actin Cytoskeleton. Curr Biol 2016, 26:1473-1479.

51. Jegou A, Carlier MF, Romet-Lemonne G: Formin mDia1 senses and generates mechanical forces on actin filaments. Nat Commun 2013, 4:1883. 
52. Courtemanche N, Lee JY, Pollard TD, Greene EC: Tension modulates actin filament polymerization mediated by formin and profilin. Proc Natl Acad Sci U S A 2013, 110:9752-9757.

53. Zimmermann D, Homa KE, Hocky GM, Pollard LW, De La Cruz EM, Voth GA, Trybus KM, Kovar DR: Mechanoregulated inhibition of formin facilitates contractile actomyosin ring assembly. Nat Commun 2017, 8:703.

54. Stam S, Alberts J, Gardel ML, Munro E: Isoforms Confer Characteristic Force Generation and Mechanosensation by Myosin II Filaments. Biophys $J$ 2015, 108:1997-2006.

55. Bergert M, Chandradoss SD, Desai RA, Paluch E: Cell mechanics control rapid transitions between blebs and lamellipodia during migration. Proc Natl Acad Sci U S A 2012, 109:14434-14439.

56. Roh-Johnson M, Goldstein B: In vivo roles for Arp2/3 in cortical actin organization during C. elegans gastrulation. J Cell Sci 2009, 122:39833993.

57. Langridge PD, Kay RR: Blebbing of Dictyostelium cells in response to chemoattractant. Exp Cell Res 2006, 312:2009-2017.

58. Yang Q, Zhang XF, Pollard TD, Forscher P: Arp2/3 complex-dependent actin networks constrain myosin II function in driving retrograde actin flow. $J$ Cell Biol 2012, 197:939-956.

59. Kassianidou E, Brand CA, Schwarz US, Kumar S: Geometry and network connectivity govern the mechanics of stress fibers. Proc Natl Acad Sci U S A 2017, 114:2622-2627.

60. Chanet S, Miller CJ, Vaishnav ED, Ermentrout B, Davidson LA, Martin AC: Actomyosin meshwork mechanosensing enables tissue shape to orient cell force. Nat Commun 2017, 8:15014.

61. Zaidel-Bar R, Zhenhuan G, Luxenburg C: The contractome--a systems view of actomyosin contractility in non-muscle cells. J Cell Sci 2015, 128:22092217.

62. Biro M, Romeo Y, Kroschwald S, Bovellan M, Boden A, Tcherkezian J, Roux PP, Charras G, Paluch EK: Cell cortex composition and homeostasis resolved by integrating proteomics and quantitative imaging. Cytoskeleton (Hoboken) 2013, 70:741-754.

63. Clausen MP, Colin-York H, Schneider F, Eggeling C, Fritzsche M: Dissecting the actin cortex density and membrane-cortex distance in living cells by super-resolution microscopy. J Phys D Appl Phys 2017, 50:064002.

$*$

This paper uses super-resolution microscopy to investigate the density distribution of the actin cortex. It shows that actin density is not homogenous through the cortical layer, and that a gap of 10-20 nm exists between the cortex and the plasma membrane; the size of this gap could affect the interactions between the cortex and the membrane. This paper presents one of the first insights into the nanoscale architecture of the actin cortex in live cells.

64. Beach JR, Bruun KS, Shao L, Li D, Swider Z, Remmert K, Zhang Y, Conti MA, Adelstein RS, Rusan NM, et al.: Actin dynamics and competition for myosin monomer govern the sequential amplification of myosin filaments. Nat Cell Biol 2017, 19:85-93.

65. Murugesan S, Hong J, Yi J, Li D, Beach JR, Shao L, Meinhardt J, Madison G, Wu $\mathrm{X}$, Betzig E, et al.: Formin-generated actomyosin arcs propel $\mathrm{T}$ cell 
receptor microcluster movement at the immune synapse. J Cell Biol 2016, 215:383-399.

66. Sarov M, Barz C, Jambor H, Hein MY, Schmied C, Suchold D, Stender B, Janosch S, K JV, Krishnan RT, et al.: A genome-wide resource for the analysis of protein localisation in Drosophila. Elife 2016, 5:e12068. 

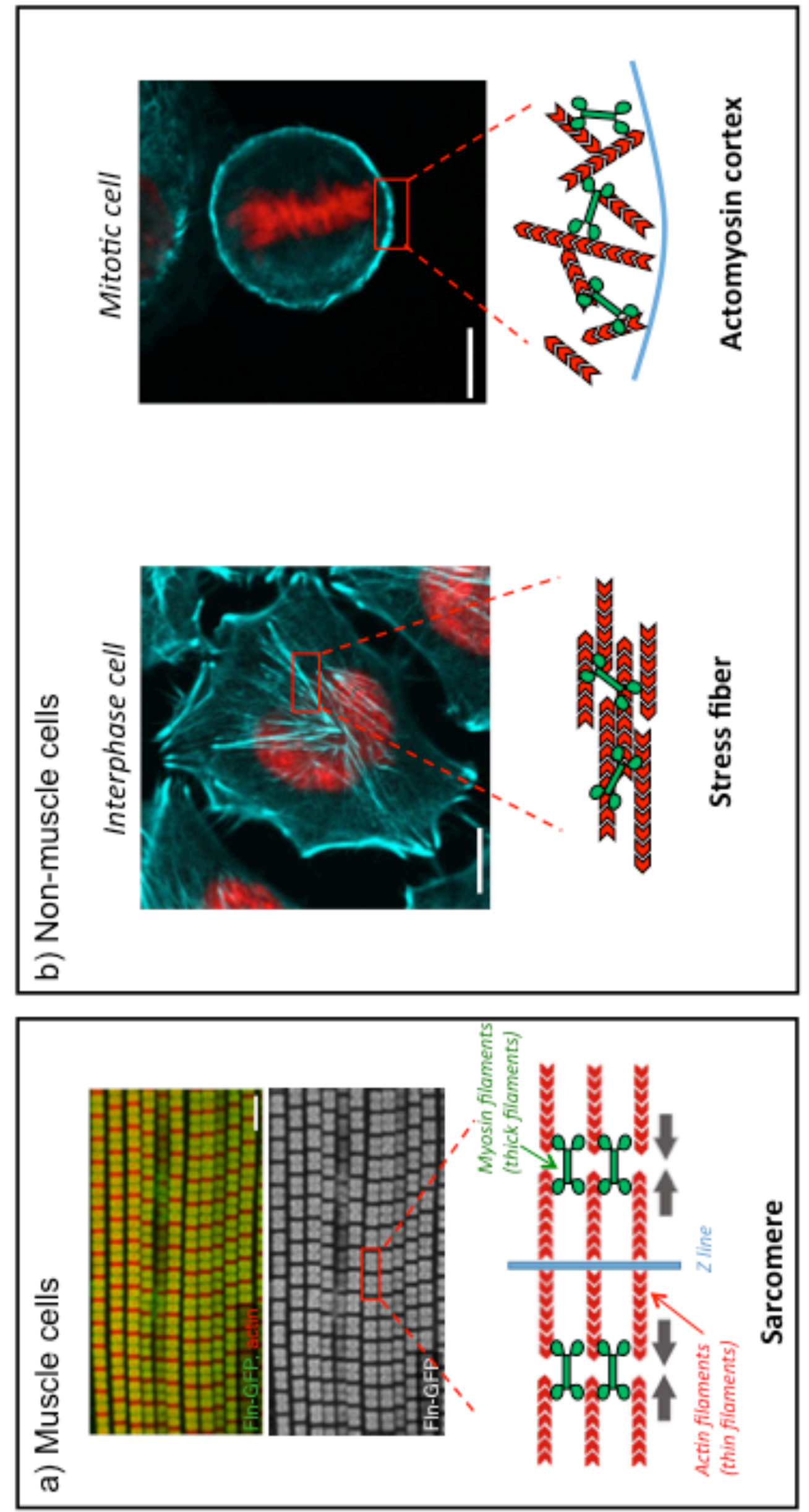


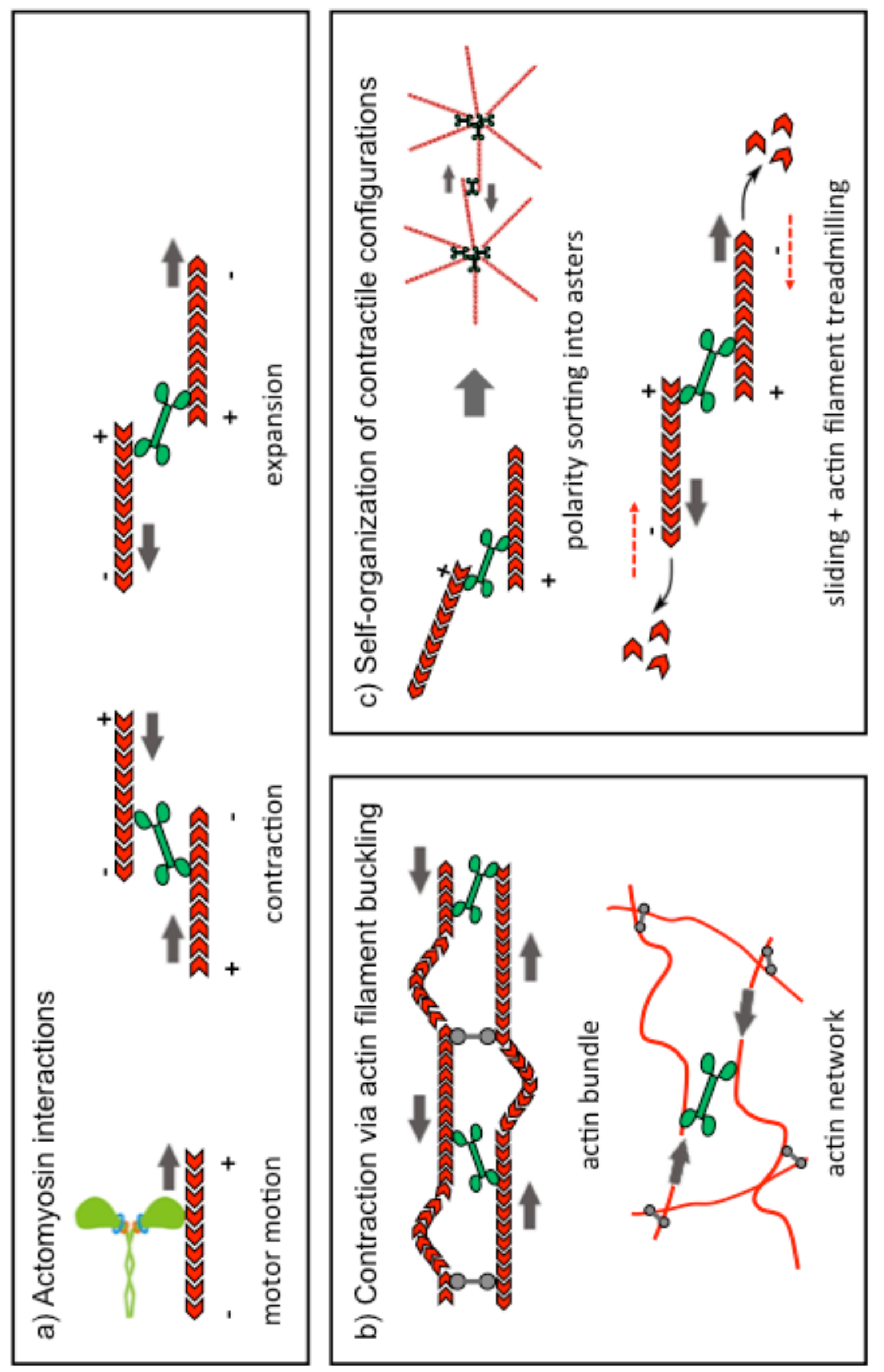

